\title{
Textura do ReCuRso Pedagógico e IMPLICAÇões EM ATIVIDADE de ENCAIXE REALIZADA POR INDIVÍDUOS COM PARALISIA CEREBRAL
}

\author{
TEXTURE OF THE PEDAGOGIC RESOURCE AND IM PLICATIONS IN FITTING \\ ACTIVITY PERFORMED BY INDIVIDUALS WITH CEREBRAL PALSY
}

\author{
Paula do Carmo PAIVA ${ }^{1}$ \\ Lígia Maria Presumido BRACCIALLI²
}

\begin{abstract}
RESUM 0: o estudo teve o propósito de verificar a influência da textura do recurso pedagógico no tempo despendido e no índice de retidão para execução de uma atividade de encaixe realizada por indivíduos com paralisia cerebral. Participaram do estudo 6 alunos com sequelas de paralisia cerebral do tipo espástica, com idade entre 7 anos e 8 meses e 28 anos e 1 mês. O participante deveria segurar o objeto e levá-lo a um ponto demarcado previamente para o encaixe e, após, retorná-lo à posição inicial. O objeto foi apresentado nas texturas lisa, intermediária e áspera, de forma aleatória. Para a coleta de dados, foi utilizada filmagem para posterior leitura pelo programa Kavideo, que possibilitou cálculos para observar o tempo despendido na tarefa e o índice de retidão. Foi realizado o estudo da normalidade das distribuições, verificada por meio do teste de normalidade de Kolmogorov-Smirnov (Teste KS) e a comparação entre as texturas lisa, intermediária e áspera, no movimento de encaixe, por meio da análise de variância de medidas repetidas. Adotou-se para todos os testes nível de significância de 5\% de probabilidade para a rejeição da hipótese de normalidade. O resultado do teste de análise de variância de medidas repetidas mostrou que não houve significância para as variáveis estudadas com o recurso apresentado nas três texturas. Porém, os dados da estatística descritiva mostraram que o encaixe do recurso com a textura lisa despendeu um menor tempo para execução da tarefa e, também, determinou um melhor desempenho com relação à trajetória do movimento.
\end{abstract}

PALAVRAS-CHAVE: Educação Especial; Educação dos deficientes físicos; Material didático.

\begin{abstract}
: the study aimed to verify how the texture of a pedagogic resource influenced the time individuals with cerebral palsy took fitting the correct piece in a puzzle. Six students with spastic type sequelae between the ages of 7 years and 8 months and 28 years and 1 month participated in the study The participants were expected to hold the puzzle piece and take it to the previously marked spot where it fit, and then take it back to its original position. The object was randomly presented in three textures: smooth, intermediate and rough. The data collected was filmed and later processed using the Kavideo program which enabled the researchers to calculate the time spent in the task and the index of correct responses. The study of normality distribution was conducted and verified using the Kolmogorov-Smirnov test for normality (KS Tests) and fitting movements were compared for smooth, intermediate and rough textures through variance analysis of repeated measures. For all tests a $5 \%$ rejection probability rate was adopted as a significant level for the normality hypothesis. The results of the variance analysis of repeated measures test showed that there was no statistical significance for the variables studied with the resource presented in the three textures. However, descriptive statistics data showed that less time was spent fitting the piece that had smooth texture during task performance and it also determined better performance related to the path of movement.
\end{abstract}

KEYWORDS: Special education; Education of people with disability; Teaching materials.

\footnotetext{
${ }^{1}$ Mestre em Educação pelo Programa de Pós-graduação em Educação - FFC - Unesp - campus de Marília - ppaivapos@yahoo.com.br

2 Docente do Programa de Pós-graduação em Educação - FFC - Unesp - campus de Maríliabracci@marilia.unesp.br - Agências financiadoras: MEC/ SEESP/ CAPES/ PROESP e CN Pq
} 


\section{INTRODUÇão}

Crianças com sequelas de paralisia cerebral (PC) que apresentam alterações motoras estão incluídas na população de alunos com deficiência física. Desta forma, essas crianças, por meio de dispositivos legais das políticas educacionais, principalmente a Resolução CNE/ CEB $\mathrm{n}$ ㄴ 2, de 11 de setembro de 2001 (BRASIL, 2001), devem ter garantido acessibilidade referente às instal ações, equipamentos emobiliários, al ém deprovimento derecursos humanos emateriais necessários, deforma a contemplar o desenvolvimento de suas capacidades físicas, cognitivas, sociais e emocionais, de acordo com suas necessidades especiais.

O ambiente escolar, principalmente a sala de aula, é um meio no qual o aluno está exposto a vários tipos de atividades pedagógicas que podem Ihe proporcionar as mais diversas experiências. Para o aluno que apresenta deficiência física, algumas dessas atividades necessitam de recursos pedagógicos adaptados para atenderem as suas necessi dades específicas e, deste modo, propiciar situações estimulantes e motivacionais que cooperem com a eficácia de seu aprendizado. 0 conceito de recurso pedagógico foi definido por Manzini (1999) como um estímulo concreto que pode ser manipulável e que possui finalidade pedagógica.

A utilização de recursos adaptados pode contribuir para aquisição de habilidades funcionais que a criança não seria capaz de realizar sem o uso destes. Além disso, a utilização desses recursos não apenas pode promover estímulos sensoriais e motores, necessários para o desenvolvimento da criança, como, ao mesmo tempo, contribuir para desenvolver os aspectos cognitivo, perceptivo, emocional e social (AUBERT, 2002). Para Teixeira, A riga e Yassuko (2003), a adaptação é um ramo da tecnologia assistiva e pode ser definida como a modificação da tarefa, do método e do meio ambiente, para promover independência e função.

Assim, uma das formas para adequar o recurso pedagógico às necessidades específicas do aluno pode ser realizada por meio de adaptações, as quais, segundo Araújo e Manzini (2001), podem ser dirigidas aos materiais pedagógicos, como lápis, caderno, livros, quadro negro, entre outros utilizados pelo aluno.

De acordo com os relatos de Manzini e Santos (2002), as adaptações com relação à textura do recurso pedagógico, são usadas para proporcionar o desenvolvimento da percepção tatilcinestésica, de forma a propiciar a vivência de várias sensações táteis, as quais podem servir de estímulo para as funções manuais presentes nas atividades do cotidiano escolar e nas atividades de vida diária. Desta forma, pequenas modificações no recurso pedagógico e, ainda, a prática e experiências repetidas, podem produzir maiores êxitos no desempenho motor desses alunos.

Os alunos com PC podem apresentar algumas dificuldades relacionadas à preensão de objetos, em virtude dos comprometimentos motores 
apresentados. Assim, algumas vezes, não adaptar as características físicas desses recursos, como formato, peso, tamanho e textura, poderá culminar no insucesso durante atividades específicas que envolvam movimentos com os membros superiores, já que estes também podem ser considerados importantes ferramentas para a exploração do meio e aquisição de novos conhecimentos.

Shumway-Cook e Woollacott (2003) destacaram a importância da percepção individual das características do objeto a ser pego para a preparação do movimento, de forma que o resultado final seja melhor, ou no mínimo, menos prejudicado. Dessa maneira, adaptações realizadas nos recursos pedagógicos usados pelo aluno com PC, quando atendem as suas necessidades específicas, podem contribuir para evitar frustração no desempenho da atividade pedagógica.

Portanto, o estudo teve o propósito de verificar se a textura do recurso pedagógico influencia no tempo despendido eno índice deretidão para a real ização de uma atividade de encaixe realizada por alunos com paralisia cerebral.

\section{MetOdologia}

\subsection{Considerações ÉtICAS}

O projeto foi aprovado pelo Comitê de Ética e pelo Conselho Diretor do Centro de Estudos da Educação e da Saúde (CEES), da Faculdade de Filosofia e Ciências - FFC, Unesp, Campus de Marília, segundo o parecer número 1413/ 2006.

\subsection{LOCAL}

A coleta dos dados foi realizada no Laboratório de Análise do Movimento (LABAM), local izado na Faculdade de Filosofia eCiências de Marília.

\subsection{Participantes}

Participaram do estudo 6 indivíduos do gênero masculino efeminino, com idade entre 7 anos e 8 meses e 28 anos e 1 mês, que apresentavam sequelas de paral isia cerebral espástica dos tipos quadriplegia e diplegia. Foram excluídos do estudo aqueles indivíduos que não possuíam diagnóstico de paralisia cerebral espástica dos tipos quadriplegia ou diplegia; apresentavam ação motora no membro superior dominante com força inferior à ação da gravidade e tinham déficit acentuado de acuidade visual.

Todos os participantes foram classificados pela escala Gross M otor Function M easure Classification System (GMFMCS), para identificar o nível de comprometimento motor (RUSSEL et al., 2002). 
No Quadro 1, visualiza-se a distribuição topográfica, o membro superior dominante, o nível de comprometimento motor segundo o GMFMCS, a idade e o gênero dos participantes.

\begin{tabular}{|l|c|c|c|c|c|}
\hline & $\begin{array}{c}\text { Distribuição } \\
\text { topográfica }\end{array}$ & $\begin{array}{c}\text { Membro } \\
\text { superior } \\
\text { dominante }\end{array}$ & $\begin{array}{c}\text { Classificação } \\
\text { GMFMCS }\end{array}$ & Gênero \\
\hline P1 & Diplegia & Direito & Nível I & 19 anos e 10 meses & Masculino \\
\hline P2 & Diplegia & Direito & Nível I & 11 anos e 3 meses & Feminino \\
\hline P3 & Quadriplegia & Direito & Nível V & 17 anos e 9 meses & Masculino \\
\hline P4 & Diplegia & Direito & Nível I & 7 anos e 8 meses & Feminino \\
\hline P5 & Quadriplegia & Esquerdo & Nível V & 14 anos e 5 meses & Masculino \\
\hline P6 & Quadriplegia & Esquerdo & Nível V & 28 anos e 1 mês & Masculino \\
\hline
\end{tabular}

Quadro 1 - Caracterização dos participantes do estudo.

\subsection{Equipamentos e materiais}

Para a realização do estudo foram utilizados: uma filmadora digital Sony H andycam, modelo DCR-HC21; fita de vídeo compatível com a filmadora; computador com placa de captura de vídeo; programa para análise demovimento Kavideo; suporte para filmadora; marcadores reflexivos; fita métrica; mobiliário adaptado; recurso pedagógico de encaixe no formato de torre.

\subsection{SituAÇão EXPERIMENTAL}

Cada participantefoi posicionado sentado na cadeira de um mobiliário adaptado, com ambos os membros superiores apoiados sobre a mesa com recorte em semicírculo no tampo, sendo realizado o ajuste da altura da mesa e do apoio para os pés para cada participante, quando necessário. O membro não utilizado na tarefa ficou apenas apoiado. O membro superior usado para a tarefa, ficou apoiado sobre a mesa com o cotovelo fletido com mão local izada no ponto inicial marcado sobre a mesa.

Para a realização do estudo foi confeccionado um recurso pedagógico adaptado do tipo encaixe. Os encaixes do recurso ficaram dispostos à frente do participante, sobre a mesa, da seguinte forma: todas as caixas foram fixadas lado a lado com espaço de $10 \mathrm{~cm}$ entre elas, no limite da distância do alcance máximo de cada participante. A atividade de encaixe foi realizada, com uma torre amarela, no formato de cilindro, que possibilitava a troca de textura do recurso. A torre amarela ficou posicionada na metade da distância entre o ponto inicial do posicionamento da mão e o alcance máximo do participante (Figura 1). 


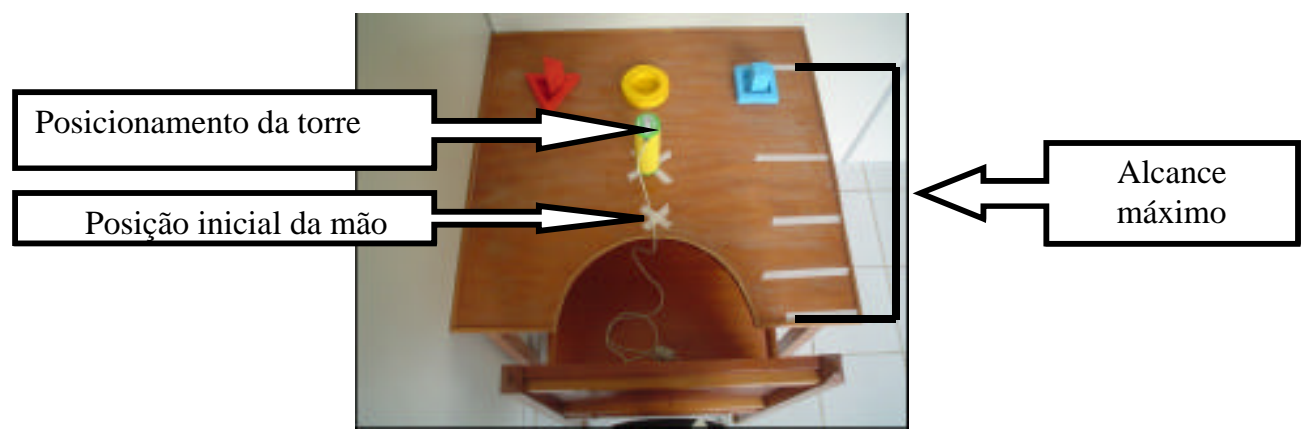

Figura 1- Posicionamento no mobiliário adaptado do recurso pedagógico utilizado no experimento.

Durante a situação experimental o recurso pedagógico foi revestido com três texturas: a) lisa: revestimento com papel verniz; b) intermediária: revestimento com papel camurça; c) áspera: revestimento com lixa com gramatura número 200.

Para determinar a ordem da utilização das três texturas - lisa, intermediária e áspera - foi realizado um sorteio prévio para cada participante e os resultados registrados em uma ficha.

Os registros foram feitos com as três texturas, lisa, intermediária e áspera, a partir do posicionamento descrito anteriormente, durante as seguintes situações: A) Preensão da torre amarela e deslocamento do membro superior dominante com a torre apreendida até a caixa amarela para o encaixe; B) A pós o encaixe, o participante deveria soltar a torre na caixa amarela (Figura 2).

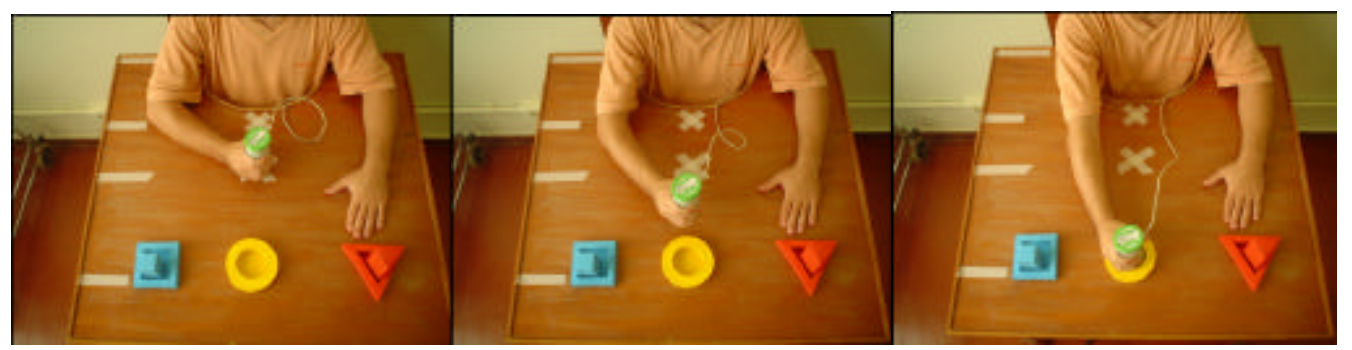

Figura 2 - Registro do movimento de encaixe real izado.

Para realizar a captura e a análise do movimento foi necessário fixar marcadores reflexivos autoadesivos sobre pontos anatômicos do corpo para posterior digitalização, a saber: 1) proeminências ósseas do acrômio; 2) o epicôndilo lateral da ulna; 3) processo estiloi de da ulna e do rádio; 4) articulações metacarpofalangeanas do polegar, dedo indicador e dedo médio. 
A tarefa solicitada foi realizada com três repetições em cada uma das três texturas, ou seja, um total de 9 repetições para cada participante, sendo realizada uma pausa de 10 segundos entre as tentativas para evitar fadiga. A troca das texturas era feita por trás do participante sem que ele visse.

\subsection{REgistro dos dAdos CINEMÁtICOS}

Para a coleta de dados a câmera digital ficou suspensa a uma altura de 1,58m do tampo da mesa, sobre a qual foram dispostos os objetos e realizada a tarefa solicitada, de forma a permitir uma visualização superior (Figura 3).

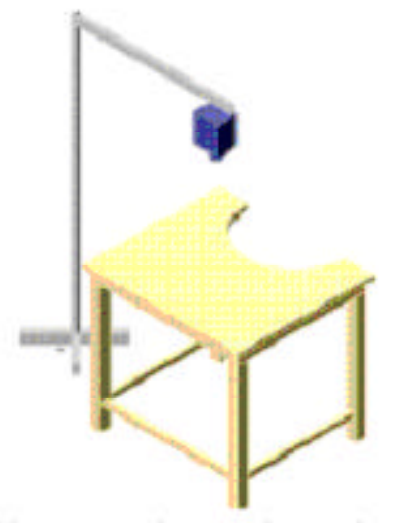

Figura 3 - Posição da filmadora com vista superior, pedestal de suporte e mesa em semicírculo.

Fonte: AUDI (2006). Estudo comparativo do comportamento motor de membro superior em encefalopatas que fazem uso de pulseira estabilizadora.

\subsection{ANÁLISE CINEMÁtica}

A análise cinemática em duas dimensões (2D) foi realizada pelo programa de análise Kavideo. Uma placa de vídeo do computador permitiu a captura das imagens por meio de uma entrada compatível com a filmadora utilizada. A simagens foram capturadas para o programa de análise do movimento Kavideo e transformadas em arquivos no formato AVI. A pós, foi realizada a formatação dos frames e conversão gráfica, por meio da digitalização realizada com o cursor do mouse sobre o centro de cada marcador. Pelo programa foram obtidos osframes ea velocidade escalar. A partir desses dados foi possível calcular: tempo despendido para a realização da tarefa em segundos (s) e o índice de retidão. $O$ índice de retidão fornece a trajetória do movimento, de forma a ilustrar o controle do mesmo (CARVALHO, 2004). 


\subsection{Análise estatística}

Em virtude da natureza dos achados os mesmos foram resumidos por meio de média e desvio-padrão. O estudo da normalidade das distribuições em estudo foi verificado por meio do teste denormalidade deK ol mogorov-Smirnov (Teste KS), e a comparação entre os grupos segundo as texturas lisa (T1), intermediária (T2) e áspera (T3), no movimento de encaixe da torre, foi realizada por meio da análise de variância de medidas repetidas e se necessário complementada por meio do teste de comparações múltiplas de Tukey (ARMITAGE; BERRY, 1997). A dotou-se para todos os testes o nível de significância de $5 \%$ de probabilidade para a rejeição da hipótese de normalidade.

\section{Resultados}

3.1 Resultados da ANÁlise do TEMPO DESPENDIDO, DURANTE O MOVIMENTO DE ENCAIXE, COM O RECURSO PEDAGÓGICO APRESENTADO EM DIFERENTES TEXTURAS

A análise descritiva e analítica dos dados do tempo despendido, durante o movimento de encaixe é apresentada na Tabela 1.

Tabela 1 - Resultados da análise do tempo despendido (s), durante o movimento de encaixe para cada textura. Teste de normalidade de Kolmogorov-Smirnov (Teste KS) e da análise de variância de medidas repetidas para a variável tempo despendido, segundo as texturas lisa (T1), intermediária (T2) e áspera (T3).

\begin{tabular}{lllllllll}
\hline Grupo & $\mathbf{n}$ & Teste KS & $\mathbf{p}$ & Média & DP & F & p & Resultado \\
\hline T1 & 6 & 0.202 & $>0.010$ & 3.245 & 1.464 & 0.961 & 0.415 & NS \\
T2 & 6 & 0.190 & $>0.010$ & 3.618 & 0.622 & & & \\
T3 & 6 & 0.191 & $>0.010$ & 4.271 & 2.812 & & & \\
\hline
\end{tabular}

Os resultados da estatística analítica mostraram que não houve diferença estatística para o movimento de encaixe.

Com relação à estatística descritiva, os resultados mostraram que, durante o movimento de encaixe, o val or mais baixo para a média foi encontrado para a textura lisa (T1), cujo valor foi de $3.245 \mathrm{~s}$, seguido do valor encontrado para a textura intermediária (T2), que foi de $3.618 \mathrm{~s}$, e para a textura áspera (T3), de $4.271 \mathrm{~s}$. Já o desvio-padrão foi de $1.464 \mathrm{~s}$ para a textura lisa (T1), $0.622 \mathrm{~s}$ para a textura intermediária (T2) e 2.812 s para a textura áspera (T3). 
3.2 RESULTADOS DA ANÁLISE DO ÍNDICE DE RETIDÃO, DURANTE O MOVIMENTO DE ENCAIXE, COM O RECURSO PEDAGÓGICO APRESENTADO EM DIFERENTESTEXTURAS

A análise descritiva eanalítica dos dados do índice deretidão, durante os movimentos de encaixe é apresentada na Tabela 2.

Tabela 2 - Resultados da análise do índice de retidão, durante o movimento de encaixe para cada textura. Teste de normalidade de Kolmogorov-Smirnov (Teste KS) e da análise de variância de medidas repetidas para a variável índice de retidão, segundo as texturas lisa (T1), intermediária (T2) e áspera (T3).

\begin{tabular}{lllllllll}
\hline Grupo & n & Teste KS & p & Média & DP & F & p & Resultado \\
\hline T1 & 6 & 0.249 & $>0.010$ & 1.751 & 0.904 & 0.939 & 0.423 & NS \\
T2 & 6 & 0.220 & $>0.010$ & 1.999 & 1.059 & & & \\
T3 & 6 & 0.262 & $>0.010$ & 1.985 & 0.914 & & & \\
\hline
\end{tabular}

A estatística analítica mostrou que não houve diferença estatística para o movimento de encaixe ( $p$ igual a 0.423 ) para as diferentes texturas.

Os resultados da estatística descritiva, durante o movimento de encaixe, mostraram que o índice de retidão cuja média apresentou valor mais baixo foi para a textura lisa (T1), com valor de 1.751. As texturas áspera (T3) e intermediária (T2) apresentaram valores bem próximos, de 1.985 e 1.999, respectivamente. Os val ores do desvio-padrão foram de 0.904 para a textura lisa (T1), 1.059 para a textura intermediária (T2) e 0.914 para a textura áspera (T3).

\section{Dıscussão}

Ainda que os resultados da estatística analítica mostrassem que não houve diferença estatística, os dados da estatística descritiva permitem observar alguns aspectos.

Durante o movimento de encaixe o maior valor encontrado da variável tempo despendido foi para a textura áspera (T3) eo menor para a textura lisa (T1).

De acordo com Shumway-Cook e Woollacott (2003), o tempo de movimento depende das demandas da tarefa em relação à acuidade e à distância e as estratégias para a programação dos movimentos, também variam, dependendo de o movimento exigir uma parada exata ou não, que requeira do indivíduo uma ação defrenagem. Assim, os resultados desse estudo para o tempo despendido anal isado sob esse ponto de vista, estão de acordo com o que relatou Shumway-Cook e Woollaccott (2003), para os quais os movimentos que exigem uma parada exata são mais lentos. Nesse estudo, essa desaceleração pode ter sido representada pelo momento em que os participantes deveriam encaixar o objeto. Ainda para Rowe (2001), outra explicação para movimentos mais lentos 
é que a desaceleração permitiria mais tempo para usar a realimentação visual e cinestésica, que no estudo podeter sido real izada para que o partici panteajustasse sua ação de acordo com a variação das texturas. Ou seja, os participantes podem ter usado as informações visual e cinestésica, proporcionadas pelas texturas do objeto a fim de ajustar o movimento realizado.

No estudo de Hung, Charles e Gordon (2004), os autores relataram que, a criança com paralisia cerebral hemiplégica, ao não utilizar estratégias compensatórias para a execução do movimento, apresenta um tempo maior para execução da tarefa que as crianças com desenvolvimento normal. Isso porque, para as crianças com PC, frente às dificuldades motoras por elas apresentadas, as atividades manuais são complexas e apresentam componentes assimétricos para executá-las. Dados semelhantes também foram encontrados no estudo de Eliasson e Gordon (2000), em que os resultados indicaram que as crianças com paralisia cerebral hemiplégica foram mais lentas ao executar uma tarefa manual que as crianças com desenvolvimento normal.

Também no estudo de Chang et al. (2005), os resultados apontaram que as crianças com paralisia cerebral do tipo espástica, sob a condição de al ta precisão do alcance, apresentaram um tempo de movimento prolongado, comparado com as crianças normais. Para os autores, a unidade de tempo foi uma importante variável cinemática para quantificar a interferência da espasticidade sobre o alcance.

Nesse estudo, também pode ser considerado que, em virtude da espasticidade dos participantes, os músculos flexores de cotovelo podem ter apresentado uma resistência a ser vencida para a execução do movimento de encaixe, evidenciada por uma execução mais lenta, demonstrada pel os resultados da variável tempo despendido.

Durante a distância percorrida pela mão ao alcançar, apreender e encaixar a torre foi percorrido um trajeto. Esse trajeto pode ter sido realizado o mais próximo de uma linha reta ou não. No caso de maior proximidade com uma linha reta, melhor desempenho da execução do movimento e, ao contrário, pior desempenho. Isso porque, o índice de retidão, determinado pela diferença entre o valor da distância percorrida pela mão e o desl ocamento real do percurso, fornece a trajetória do movimento, de forma a ilustrar o controle do mesmo (CARVALHO, 2004).

A pesar dos resultados da estatística analítica terem mostrado que não houve diferença estatística para o índice de retidão, com o recurso apresentado nas três texturas usadas nesse estudo, os dados da estatística descritiva nos permitem observar alguns aspectos.

Durante a execução da tarefa, o melhor desempenho foi realizado com a textura lisa (T1). 
Pode ser que a textura influencie na trajetória do movimento e, consequentemente, no desempenho deste, já que o movimento está sujeito ao conjunto de todas essas variáveis e o seu resultado dependente da harmonia destes fatores. Para Brandão (1984), o início, a duração e a intensidade das contrações dos diferentes grupos musculares devem ser sinérgicos, associados e coordenados de tal modo que o deslocamento da mão no espaço seja uma linha contínua e harmoniosa e não executada segundo um trajeto quebrado, o que aconteceria se estes movimentos fossem isolados e sucessivos, cada um a seu tempo. Ainda para o autor, informações como peso, consistência, aspereza e temperatura são de grande importância para a escolha do padrão de preensão a ser usado e da atividade e orientação que a mão deverá tomar ao tocar o objeto, pois, a cooperação da sensibilidade proprioceptiva, do tato e da visão contribui para que a aproximação possa ser executada de modo eficiente.

De acordo com Shumway-Cook e Woollaccott (2003), normalmente, os ângulos das articulações do cotovelo e do ombro mudam de uma forma muito suave e em ritmo sincronizado, buscando produzir um movimento suave de alcance, com uma trajetória consideravelmente reta da mão. Para as autoras, nos pacientes com comprometimentos neurais, os movimentos de alcance são caracterizados pela descoordenação multiarticular, que leva a trajetórias anormais do movimento. Isso pôde ser mostrado nesse estudo, já que o índice de retidão para os seis participantes apresentou-se com um valor alto, denunciando um desempenho deficitário com relação à trajetória do movimento.

Em relação à importância de fatores intrínsecos e extrínsecos para contribuição ao movimento, Sugden (2000), afirmou que estes têm efeito sobre o alcance e preensão de crianças com paralisia cerebral hemiplégica, os quais proporcionam informações para que a criança realize adequações em seus movimentos. Para Kudoh et al. (1997) e Watson e Jakobson (1997), o componente de transporte utiliza a informação visual a respeito das propriedades extrínsecas de um objeto, como o local ea orientação do mesmo, as quais trazem informações para permitir que a mão alcance o objeto. Segundo Bradshaw et al. (2004), as informações visuais são muito importantes por duas razões: para especificar as propriedades do objeto e, dessa forma, contribuir para o pré-planejamento do transporte e componentes da preensão, e, para monitorar o desempenho do al cance e ajustes necessários para um resultado satisfatório. Já os autores Choi e Mark (2004), afirmaram que o al cance e a preensão não são apenas influenciados pela distância que o objeto a ser alcançado e apreendido localiza-se no espaço ou, ainda, pelo tamanho e peso do objeto, mas, também pela força que será necessária para o desempenho de tal execução.

Desta forma, o uso de texturas no recurso pedagógico pode permitir experiências de sensações, as quais poderão ser mais favoráveis ou não na determinação de um bom desempenho do movimento executado. N esse estudo, a textura lisa (T1) foi a que possi bilitou um movimento executado com maior precisão 
e, consequentemente, melhor desempenho durante a trajetória do movimento realizado pela mão, embora, ainda assim, esses participantes obtiveram valores maiores que 1, valor esse que caracteriza-se como desempenho ideal.

\section{CONCLUSÃo}

O estudo comparativo do comportamento motor de membros superiores em indivíduos com paralisia cerebral espástica, dos tipos diplegia e quadriplegia, durante uma atividade de encaixe do recurso pedagógico, apresentado em três diferentes texturas, mostrou que os movimentos realizados com a variação das texturas apresentaram:

Menor tempo despendido para a realização da tarefa com o recurso apresentado pela textura lisa (T1), mostrando maior eficiência;

Menor índice de retidão encontrado para a textura lisa (T1), demonstrando um melhor desempenho dos participantes com relação à trajetória do movimento executado durante a realização da tarefa solicitada.

\section{ReferênCIAS}

ARAÚJO, R. C. T.; MANZINI, E.J. Recursos na escolarização do al uno com deficiência física. In: MANZINI, E. J. (Org.). Linguagem, cognição eensino do aluno com deficiência. Marília: Unesp - Marília - Publicações, 2001. p. 1-11.

ARMITA GE, P; BERRY, G. Estadística para la investigación biomédica. 3 ed. Madrid: Harcourte Brace, 1997.

AUBERT, E. K. Equipamentos adaptativos para crianças com incapacidade física. In: TECKLIN, J. S. Fisioterapia Pediátrica. 3ed. Porto Alegre: Artmed, 2002. p. 32-45.

AUDI, M. Estudo comparativo do comportamento motor de membro su perior em encefalopatas que fazem uso de pulseira estabilizadora. 2006. 112f. Dissertação (M estrado). FaculdadedeFilosofia eCiências, Universidade Estadual Paulista, Marília.

BRADSHAW, M. F. et al. Theeffects of a premovement delay on the kinematics of prehension in middlechildhood. H uman M ovement Science, v. 23, p. 771-784, 2004.

BRANDÃ O,J. S. D esenvolvimento Psicomotor da M ão. Rio de Janeiro: Enelivros, 1984.

BRA SI L. M inistério da Educação. Secretaria deEducação Especial. D iretrizes nacionais para a educação especial na educação básica. Brasília: MEC/ SEESP, 2001.

CARVALHO, R. P. A influência da postura corporal no movimento dealcance manual em lactentes de 4 meses de vida. 2004. 117 f. Dissertação (M estrado) - Centro de ciências biológicas e da saúde, UniversidadeFederal deSão Carlos, São Carlos.

CHANG, J-J. et al. Kinematical measure for spastic reaching in children with cerebral palsy. Clinical Biomechanics, v. 20, p. 381-388, 2005. 
CHOI, H. J.; MARK, L. S. Scaling affordances for human reach actions. H uman M ovement Science, v. 23, p. 785-806, 2004.

ELIASSON , A.; GORDON , A. M. Impaired forcecoordination during object rel easein children with hemi plegic cerebral palsy. D evelopmental M edicineand Child N eurology, v. 42, p. 228-234, 2000.

HUNG, Y.; CHARLES, J.; GORDON , A. M. Bimanual coordination during a goal-directed task in children with hemiplegic cerebral palsy. D evelopmental $M$ edicineand $C$ hild $N$ eurology, v. 46, p. 746-753, 2004.

$\mathrm{KUDOH}, \mathrm{N}$. et al. A n analysis of spatiotemporal variability during prehensi on movements: effects of object sizeand distance. Experimental Brain Research, v. 117, p. 457-464, 1997.

MANZINI, E. J. Recursos pedagógicos para o ensino de alunos com paralisia cerebral. M ensagem da APAE, v. 36, n. 84, p. 17-21, 1999.

MANZINI, E. J.; SANTOS, M. C. F. Portal de ajudas técnicas para educação: equipamento e material pedagógico para educação, capacitação erecreação da pessoa com deficiência física: recursos pedagógicos adaptados. Brasília: MEC: SEESP, 2002, fascículo 1, 56p.: il.

ROWE, P. J. Parâmetros de mensuração. In: DURWARD, B. R.; BAER, G. D.; ROWE, P. J. M ovimento funcional humano: mensuração e análise. São Paulo: Manole, 2001. p. 13-33.

RUSSEL, D. J. et al. C. Gross motor function measure (GM FM 66 \& GM FM 88) user's manual . London: Mackeith Press, 2002.

SHUMWAY-COOK, A; WOOLLACOTT, M. H. ControleM otor. São Paulo: Manole, 2003.

SUGDEN, D. Dynamic coupling: intrinsic and extrinsic influences on reaching and grasping in children with hemipl egic cerebral palsy. R evista P aulista deE ducação Física. São Paulo, v. 3, p. 24-28, 2000.

TEIXEIRA, E.; ARIGA, M. Y.; YASSUKO, R. A daptações. In:TEIXEIRA, E.; SAURON, F. N.; SANTOS, L. S. B.; OLIVEIRA, M. C. Terapia ocupacional na reabilitação física. São Paulo: Roca, 2003. p. 129-173.

WATSON, M. K.; JAKOBSON, L. S. Timeto contact and the control of manual prehension. Experimental Brain Research, v. 117, p. 273-280, 1997.

Recebido em: 08/ 09/ 2008

Reformulado em: 16/ 06/ 2009

Aprovado em: 30/ 06/ 2009 